\title{
Enhanced Extreme Ultraviolet Lithography Mask Inspection Contrast Using Fabry-Perot Type Antireflective Coating
}

\author{
Hsu-Chun Cheng, Hsuen-Li Chen ${ }^{1, *}$, Tsung-Shine $\mathrm{KO}^{2}$, Lee-Jene LAI ${ }^{3}$, Fu-Hsiang Ko and Tieh-Chi ChU ${ }^{2}$ \\ National Nano Device Lab., Hsinchu, Taiwan, R. O. C. \\ ${ }^{1}$ Department of Materials Science and Engineering, National Taiwan University, Taiwan, R. O. C. \\ ${ }^{2}$ National Tsing Hua University, Hsinchu, Taiwan, R. O. C. \\ ${ }^{3}$ National Synchrotron Radiation Research Center, Hsinchu, Taiwan, R. O. C.
}

(Received October 31, 2003; revised January 9, 2004; accepted January 28, 2004; published June 29, 2004)

\begin{abstract}
In this paper, we describe two strategies for solving the low-contrast problem during extreme ultraviolet lithography (EUVL) mask inspection processes. One is the use of single-layer antireflective coating (ARC), and the other is the use of Fabry-Perottype ARC. The materials of ARC are the same as those of buffer layers, such as $\mathrm{SiO}_{2}$ and $\mathrm{Si}_{3} \mathrm{~N}_{4}$, which are easy to fabricate. Contrast can be increased up to $>95 \%$ by adding ARC. For both absorbers, Single-layer $\mathrm{Si}_{3} \mathrm{~N}_{4} \mathrm{ARC}$ and $\mathrm{Si}_{3} \mathrm{~N}_{4}$-based FabryPerot-type ARC show better performances than $\mathrm{SiO}_{2}$ ARC. Both types of ARC maintain a high contrast at $40 \%$ with a large thickness variation even until $\pm 40 \%$. Moreover, the top absorber in Fabry-Perot-type ARC has good conductivity that can eliminate electrical distortion, which is caused by electron charging during e-beam direct writing. The Fabry-Perot-type ARC structure has better contrast and thickness variation tolerance than the single-layer ARC structure. The film materials in FabryPerot structure can also be used for various absorber and dielectric materials. [DOI: 10.1143/JJAP.43.3703]
\end{abstract}

KEYWORDS: extreme ultraviolet lithography, absorber, buffer layer, antireflective coatings, Fabry-Perot

\section{Introduction}

Extreme ultraviolet lithography is one of the leading candidates for patterning semiconductor devices for sub$50 \mathrm{~nm}$ generations. ${ }^{1)}$ In recent years, a wide range of absorber and buffer layer materials have been evaluated as potential absorber materials for extreme ultraviolet lithography (EUVL) mask applications. ${ }^{2)} \mathrm{TaN}$ and $\mathrm{Cr}$ are the leading choices for absorber materials. $\mathrm{Si}_{3} \mathrm{~N}_{4}$ and $\mathrm{SiO}_{2}$ have been widely used as buffer layers. 3 )

$\mathrm{TaN}$ and $\mathrm{Cr}$ exhibit characteristics as absorbers at the EUV wavelength $(13.4 \mathrm{~nm})$ but have a high reflectance at the deep ultraviolet (DUV) inspection wavelength $(257 \mathrm{~nm}$ or $365 \mathrm{~nm})$. The high reflectance from the absorber stack decreases the contrast between multilayer stacks during inspection. Therefore, increasing the inspection contrast by decreasing reflectance from the absorber stack at the inspection wavelength is essential.

Traditionally, a mask is fabricated by electron beam direct writing. Electrons intrinsically accumulate on the incident substrate that causing following electron deflection. The problem will become more serious in the current EUVL mask fabrication than in past fabrication when the feature size was less than $200 \mathrm{~nm}$. To avoid pattern distortion, the substrate should have good conductivity. General singlelayer antireflective coatings (ARCs), which are dielectric materials with poor conductivity, are not suitable substrates in e-beam direct writing.

In this paper, we will demonstrate two simple ARC structures the use of which enables absorber stacks to meet the exposure and inspection requirements simultaneously. The thickness of ARC was optimized to minimize the reflectance at $257 \mathrm{~nm}$. We also considered solving the charging effect caused by electron accumulation using FabryPerot-type ARC. The total thickness of the absorber stacks were maintained below $150 \mathrm{~nm}$ to meet the stack height requirement thus preventing the geometric shadow effect.

*E-mail address: hlchen@ndl.gov.tw
Table I. Optical constant of EUVL mask materials at $257 \mathrm{~nm}$ wavelength.

\begin{tabular}{lcl}
\hline & $\mathrm{n}$ & \multicolumn{1}{c}{$\mathrm{k}$} \\
\hline $\mathrm{Si}$ & 1.8411 & 2.8374 \\
$\mathrm{Mo}$ & 1.7170 & 3.7458 \\
$\mathrm{SiO}_{2}$ & 1.5041 & 0 \\
$\mathrm{Si}_{3} \mathrm{~N}_{4}$ & 2.2475 & 0.0023 \\
$\mathrm{TaN}$ & 2.4967 & 1.5257 \\
$\mathrm{Cr}$ & 1.3776 & 1.9878 \\
\hline
\end{tabular}

$\lambda=257 \mathrm{~nm}$

\section{Simulation}

For all calculations, we used a commercial thin-film design program (SCI Film Wizard). The optical constants of molybdenum (Mo), silicon ( $\mathrm{Si})$, tantalum nitride (TaN), chrome $(\mathrm{Cr})$, silicon dioxide $\left(\mathrm{SiO}_{2}\right)$, and silicon nitride $\left(\mathrm{Si}_{3} \mathrm{~N}_{4}\right)$ at EUV and DUV wavelengths from the Henke table $^{4)}$ were imported to this program. Table I lists the optical constants of EUVL mask materials in the 257-nminspection-wavelength regions.

For characterizing reflectance behavior, we assume multilayer (ML) coatings consisting of 40-layer pairs of Mo and Si with layer thicknesses of 2.8 and $4.1 \mathrm{~nm}$, respectively. ${ }^{5)}$ The Mo/Si ML coatings were deposited on a low-thermalexpansion material (LTEM) substrate and then covered with buffers and absorbers by the PVD method. We assume that the thickness of buffer was approximately $60 \mathrm{~nm}$ to meet the requirement of the focused ion beam (FIB) repair stage. The thickness of the absorber was $60 \mathrm{~nm}$ to achieve a low reflectance $(<0.1 \%)$ at an exposure wavelength with and without ARC.

\section{Results and Discussion}

To simplify discussion, we obtain the same reflectance from ML stacks at the repair and final stages by calculating reflectance from the ML stack with different buffer layer thicknesses. As shown in Fig. 1, the reflectance from the ML 


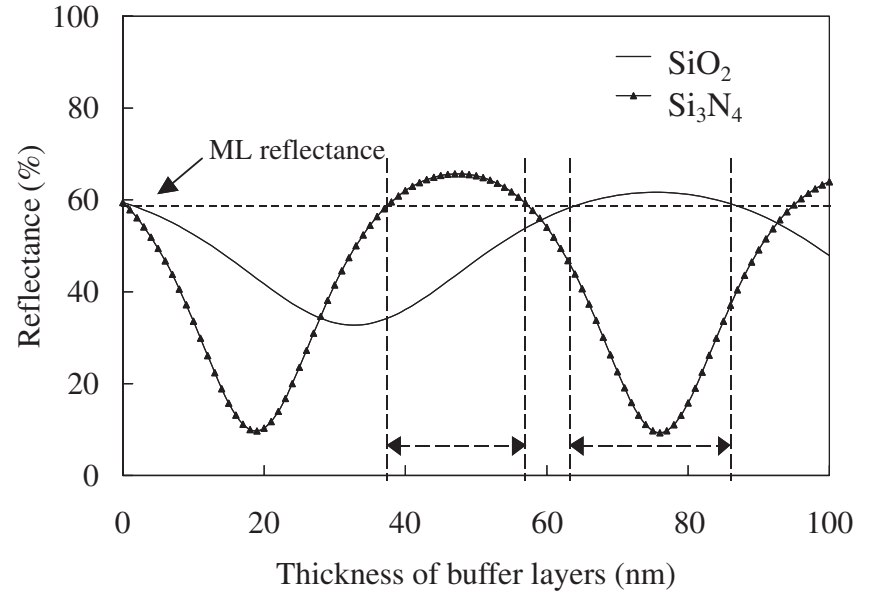

Fig. 1. Reflectance from ML mirror and different thicknesses of buffer layers.

stack is about $60 \%$ at $257 \mathrm{~nm}$. Here, we design the ARC to increase contrast by decreasing the reflectance from the absorber stacks. Therefore, the reflectance from the ML stack with a buffer should be higher or equal to $60 \%$. According to this viewpoint, the thickness of ARC should be chosen as 38 to $58 \mathrm{~nm}$ for $\mathrm{Si}_{3} \mathrm{~N}_{4}$, and 64 to $85 \mathrm{~nm}$ for $\mathrm{SiO}_{2}$. However, through the considerations of stack thickness and FIB repair requirement, an etching selectivity of the absorber to the buffer is about 1 , a buffer layer thickness of about $60 \mathrm{~nm}$ are necessary. The reflectance at such buffer layer thickness is almost the same as the final-stage buffer layer thickness. Hence, the following results and discussions focus only on the final-stage inspection, in which the reflectance is about $60 \%$.

\subsection{Single-layer ARC}

Figure 2 shows the diagram of the single-layer ARC structure. By common consideration, we chose only $\mathrm{SiO}_{2}$

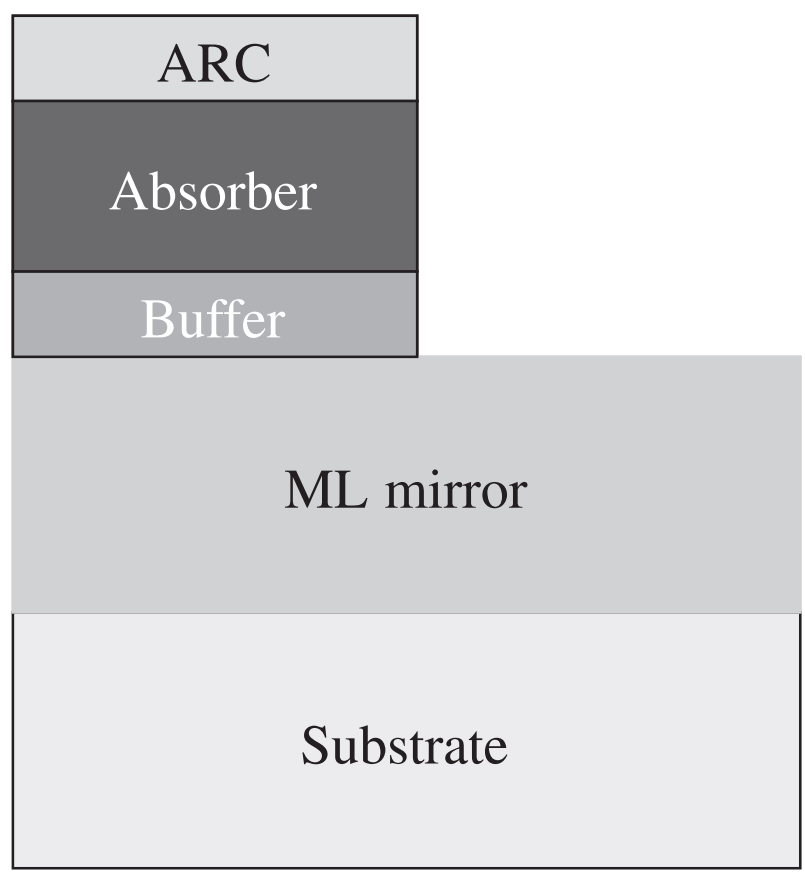

Fig. 2. Diagram of single-layer antireflective coating structure.

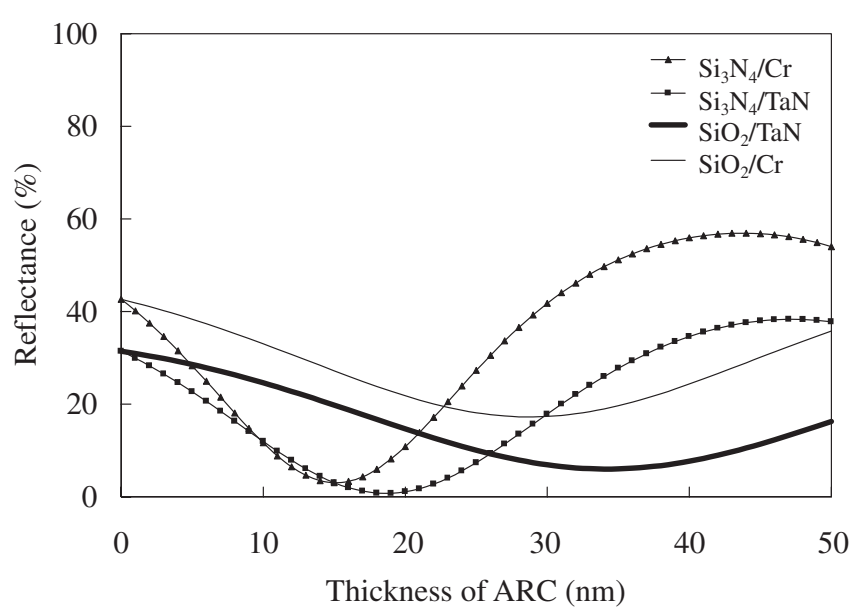

Fig. 3. Reflectance from absorber stacks with single-layer ARC at $257 \mathrm{~nm}$ inspection wavelength.

and $\mathrm{Si}_{3} \mathrm{~N}_{4}$ in our simulations. Of course, other materials such as $\mathrm{SiON}, \mathrm{Ta}_{2} \mathrm{O}_{5}$, and $\mathrm{Cr}_{2} \mathrm{O}_{3}$ could also be used for ARC. Here, we selected the same ARC material with a buffer to simplify the blank mask fabrication process. Figure 3 shows the reflectance from absorber stacks with different ARC thicknesses. As shown in the figure, the reflectance from the absorber stack become minimum after capping $\mathrm{Si}_{3} \mathrm{~N}_{4}$ and $\mathrm{SiO}_{2}$ of different thicknesses. The reflectance from thin $\mathrm{Si}_{3} \mathrm{~N}_{4}$-ARC-capped absorbers is lower than that from thick $\mathrm{SiO}_{2}$-ARC-capped ones. By adding $<20 \mathrm{~nm} \mathrm{Si}_{3} \mathrm{~N}_{4}$, the reflectance from the $\mathrm{Cr}$ absorber could be decreased to $3 \%$ and that from the $\mathrm{TaN}$ absorber to $1 \%$. It is clearly seen that $\mathrm{Si}_{3} \mathrm{~N}_{4}$ has better performance than $\mathrm{SiO}_{2}$ in terms of reflectance and total thickness.

From previous discussions, the optimum thickness of single-layer ARC was determined. However, it is difficult to produce perfect ARC in actual situations. What of the exact thickness could not be deposited, and a slight inaccuracy existed. The variation of single-layer ARC thickness causes contrast degradation under most conditions but still satisfies the inspection contrast threshold, which should be at least $40 \%$. However, in the worst case, for the $\mathrm{SiO}_{2}$-capped $\mathrm{Cr}$ absorber, the contrast is still higher than $40 \%$ when the ARC thickness varied from $\pm 40 \%$ ( $\pm 11.6 \mathrm{~nm})$, as shown in Fig. 4 . In the best case, for the $\mathrm{Si}_{3} \mathrm{~N}_{4}$-capped $\mathrm{TaN}$ absorber, the contrast is still higher than $40 \%$ when the ARC thickness varied from $\pm 80 \%( \pm 15.2 \mathrm{~nm})$. By controlling $\mathrm{Si}_{3} \mathrm{~N}_{4}$ ARC thickness well, the contrast can be from $\mathrm{TaN}$ and $\mathrm{Cr}$ raised to $97.5 \%$ and $90 \%$, respectively. These should be acceptable values for all existing equipment.

Both $\mathrm{Si}_{3} \mathrm{~N}_{4}$ and $\mathrm{SiO}_{2}$ could be used to upgrade the contrast even for $\mathrm{TaN}$ or $\mathrm{Cr}$ absorbers. From the optical viewpoint, they are almost the flawless materials. However, there is a fatal drawback, that is, conductivity. To generate perfect antireflective structures, the idea of Fabry-Perot-type ARC was proposed.

\subsection{Fabry-Perot-type ARC}

Figure 5 shows a Fabry-Perot-type ARC structure, which comprise a dielectric layer and a thin absorber layer. In general, the mask layer should have good electrical conductivity for e-beam writing in order to prevent writing 


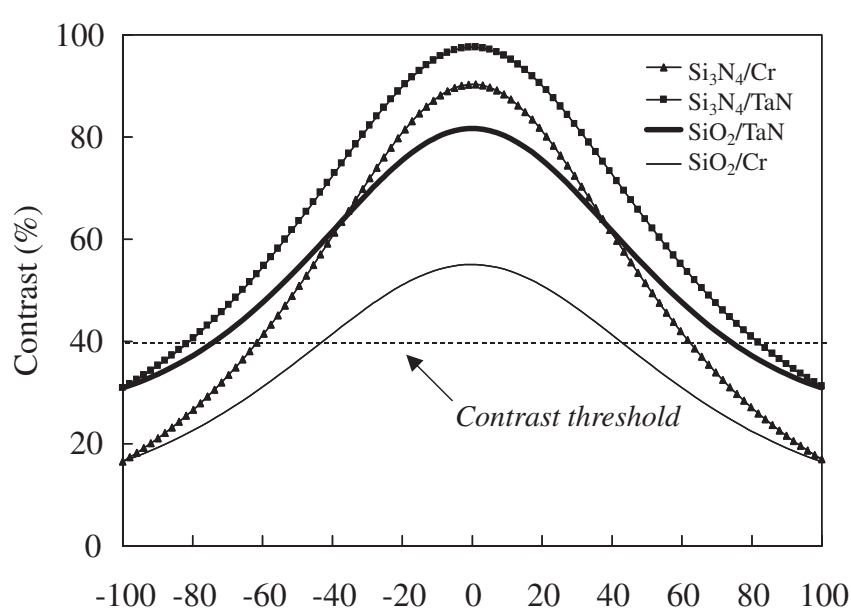

Variation from Optimum Thickness (\%)

Fig. 4. Thickness variation tolerance of single-layer ARC.

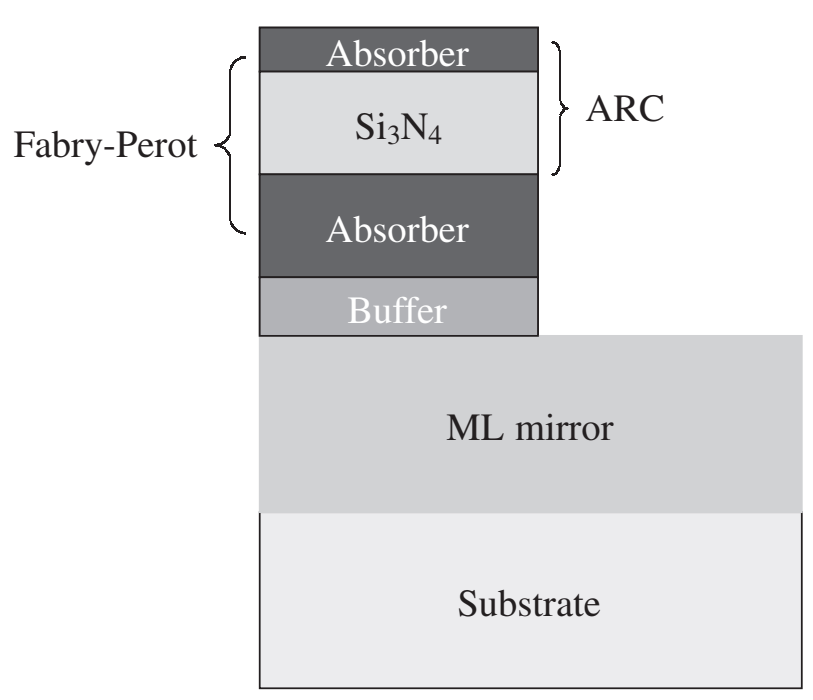

Fig. 5. Diagram of a Fabry-Perot-type antireflective coating structure.

errors due to charging effects. In the Fabry-Perot structure, the top absorber layer can prevent charge accumulation during e-beam writing. By controlling the thickness of the dielectric layer, we can easily tune the minimum reflection region to the desired inspection wavelength. The reflectances at $257 \mathrm{~nm}$ are shown in Fig. 6. We fixed the thickness of the top absorber layer at several $\mathrm{nm}$ and change the thickness of the bottom dielectric layer to evaluate reflectance from the stack. The total absorber thickness in the stack was fixed at $60 \mathrm{~nm}$ and it is easy to achieve both low EUV reflectance and high DUV contrast by controlling the $\mathrm{Si}_{3} \mathrm{~N}_{4}$ or $\mathrm{SiO}_{2}$ thickness well. As shown in Fig., $\mathrm{SiO}_{2}$ has the same performance as $\mathrm{Si}_{3} \mathrm{~N}_{4}$ in terms of reflectance, but is thicker. Reflectance decreases to $<1 \%$ in all cases when the thicknesses of $\mathrm{Si}_{3} \mathrm{~N}_{4}$ and $\mathrm{SiO}_{2}$ were about $20 \mathrm{~nm}$ and $30 \mathrm{~nm}$, respectively. All contrasts are higher than $95 \%$.

Similarly, we focus on the contrast decrease caused by varying ARC thickness. Figure 7 shows the relationship between the thickness of $\mathrm{Si}_{3} \mathrm{~N}_{4}$-based Fabry-Perot-type ARC and contrast. Both the top absorber and bottom dielectric layers of Fabry-Perot-type ARC show good thickness variation tolerance.

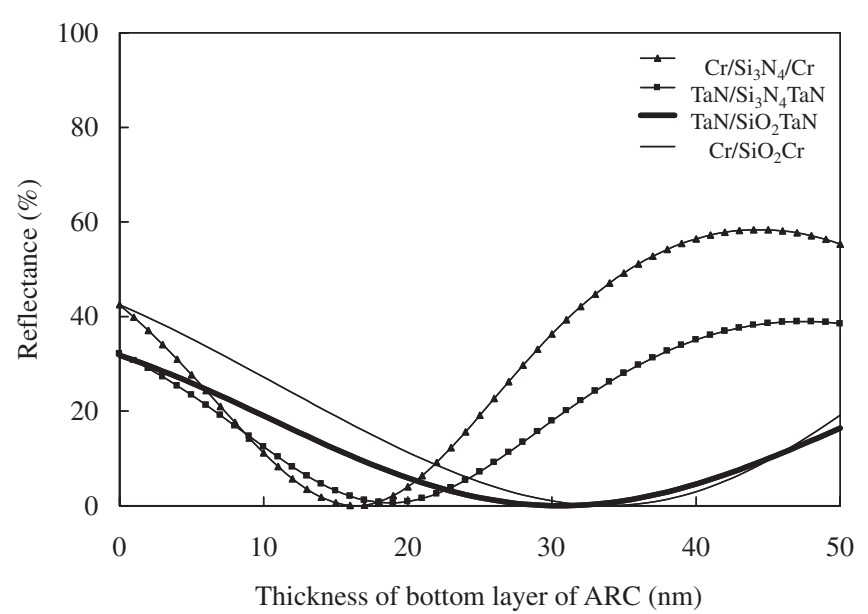

Fig. 6. Reflectance from absorber stacks with a Fabry-Perot-type ARC at $257 \mathrm{~nm}$ inspection wavelength.

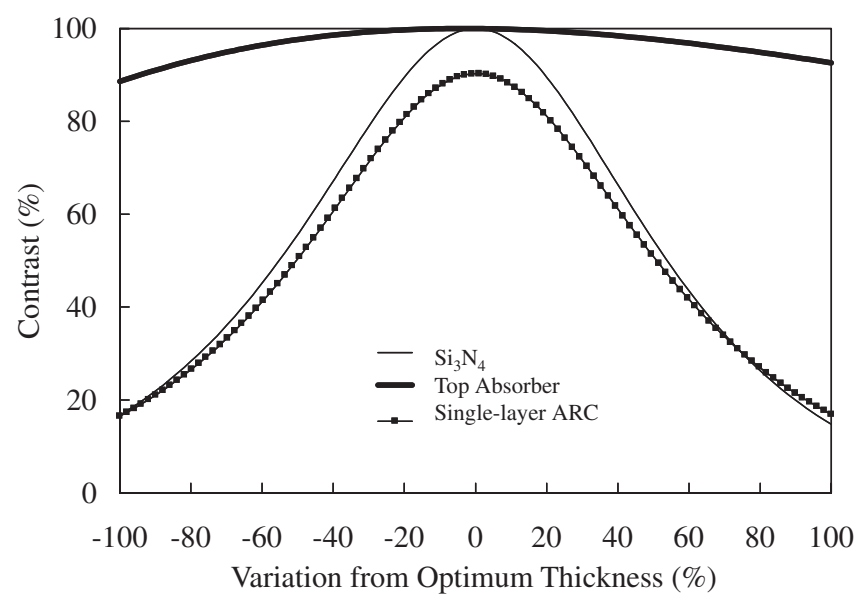

Fig. 7. Comparison of contrast and thickness tolerance between singlelayer and Fabry-Perot-type ARCs.

\subsection{Comparisons of single-layer ARC and Fabry-Perot-} type ARC

As shown in Fig. 7, $\mathrm{Cr}$ is the absorber and $\mathrm{Si}_{3} \mathrm{~N}_{4}$ is the single-layer ARC. By comparing the thickness tolerance and performance of these two ARC structures, Fabry-Perot-type ARC shows better performance than single-layer ARC. As summarized in Table II, the contrasts without adding ARC were $30.9 \%$ and $16.5 \%$ from $\mathrm{TaN}$ and $\mathrm{Cr}$ absorbers, respectively. It is difficult to recognize pattern defects using the inspection equipment at this low contrast level. After capping a $\mathrm{SiO}_{2}$ single-layer ARC, the contrasts were raised to $81.7 \%$ and $55.1 \%$. These are acceptable inspection thresholds but not sufficiently good. Thus, we raised the

Table II. Contrasts (\%) of traditional EUVL mask with/without ARC at $257 \mathrm{~nm}$ wavelength.

\begin{tabular}{|c|c|c|c|c|c|}
\hline & \multirow{3}{*}{ Without ARC } & \multicolumn{4}{|c|}{ With ARC } \\
\hline & & \multicolumn{2}{|c|}{ Single-layer } & \multicolumn{2}{|c|}{ F-T type } \\
\hline & & $\mathrm{SiO}_{2}$ & $\mathrm{Si}_{3} \mathrm{~N}_{4}$ & $\mathrm{SiO}_{2}$ & $\mathrm{Si}_{3} \mathrm{~N}_{4}$ \\
\hline $\mathrm{TaN}$ & 30.9 & 81.7 & 97.5 & $>99$ & 98 \\
\hline $\mathrm{Cr}$ & 16.5 & 55.1 & 90.3 & $>99$ & $>99$ \\
\hline
\end{tabular}


Table III. Total thickness (nanometer) of absorber stacks with/without ARC.

\begin{tabular}{lcccccc}
\hline & & \multicolumn{4}{c}{ With ARC } \\
\cline { 3 - 4 } & \multirow{2}{*}{ Without ARC } & \multicolumn{2}{c}{ Single-layer } & & \multicolumn{2}{c}{ F-T type } \\
\cline { 3 - 4 } \cline { 6 - 7 } & & $\mathrm{SiO}_{2}$ & $\mathrm{Si}_{3} \mathrm{~N}_{4}$ & & $\mathrm{SiO}_{2}$ & $\mathrm{Si}_{3} \mathrm{~N}_{4}$ \\
\hline $\mathrm{TaN}$ & 120 & $34 / 120$ & $19 / 120$ & & $31 / 120$ & $19 / 120$ \\
$\mathrm{Cr}$ & 120 & $29 / 120$ & $15 / 120$ & & $34 / 120$ & $16 / 120$ \\
\hline
\end{tabular}

contrasts to $97.5 \%$ and $90.3 \%$ by capping $\mathrm{Si}_{3} \mathrm{~N}_{4}$ single-layer ARC. These results seem to be better than those of $\mathrm{SiO}_{2}-$ capped ones.

Furthermore, as shown in Table III, the total thickness of the $\mathrm{SiO}_{2}$-capped absorber stacks were too large to conform to the rule that the thickness should as small as possible. On the other hand, the $\mathrm{Si}_{3} \mathrm{~N}_{4}$-capped absorber stack fulfilled the requirement.

Similarly, the contrasts could be raised to more than $95 \%$ by adding Fabry-Perot-type ARC in all situations. The thicknesses of stacks were almost the same as that for singlelayer ARC that did not increase much. $\mathrm{Si}_{3} \mathrm{~N}_{4}$ performs better than $\mathrm{SiO}_{2}$ in a comprehensive survey. Thickness variation tolerance was as large as that of the single-layer ones. In addition, Fabry-Perot-type ARC has good conductivity to eliminate the charging effect during e-beam writing.

\section{Conclusions}

Two types of ARC were demonstrated by simulation. Single-layer ARC has an acceptable contrast that meets equipment requirements. Fabry-Perot-type ARC not only shown excellent contrast but also good conductivity. It can eliminate the electron charging effect thereby preventing pattern distortion during mask fabrication and raising contrast during the inspection process simultaneously. Thinner $\mathrm{Si}_{3} \mathrm{~N}_{4}$ had a higher contrast than thicker $\mathrm{SiO}_{2}$. However, from the viewpoint of contrast and stack thickness that induce the geometric shadow effect, the $\mathrm{Si}_{3} \mathrm{~N}_{4}$ performed better than $\mathrm{SiO}_{2}$ in both types ARC.

1) S. D. Hector: SPIE 4688 (2002) 134.

2) P. Y. Yan, G. Zhamg, A. Ma and T. Liang: SPIE 4343 (2001) 409.

3) K. H. Smith, J. R. Wasson, P. J. S. Mangat, W. J. Dauksher and D. J. Resnick: J. Vac. Sci. \& Technol. B 19 (2001) 2906.

4) B. L. Henke, E. M. Gullikson and J. C. Davis: At. Data \& Nucl. Data Tables 54 (1993) 181.

5) S. D. Hector and P. Mangat: J. Vac. Sci. \& Technol. B 19 (2001) 2612. 